\title{
Statistically Optimized Synthesis of Silver Nanocubes from Peel Extracts of Citrus limetta and Potential Application in Waste Water Treatment
}

Priyanka Trivedi, Manish Khandelwal and Priyanka Srivastava*

School of Bio Sciences and Technology, Vellore Institute of Technology University, Tamil Nadu, India

\begin{abstract}
This work reports statistically optimized synthesis of silver nanocubes from cost effective, readily available bioreductant, in the form of peels of Citrus limetta. Effect of six variables viz. temperature, pH, volume of reductant, reaction volume, illumination, concentration of silver nitrate was examined using Placket Burman Design. Temperature, $\mathrm{pH}$ and illumination were found to significantly affect the shape and size of silver nanoparticles. Best conditions for the synthesis of silver nanocubes were $0.001 \mathrm{M} \mathrm{AgNO}_{3}$ with temperature $27^{\circ} \mathrm{C}$ and $\mathrm{pH} 6$ under dark incubation. Characterization of the synthesized nanocubes was done using UV-Vis spectroscopy, Scanning Electron Microscopy (SEM). SEM analysis showed clear synthesis of nanocubes in the range of 37-59 nm to 163-205 nm. Additionally, an investigation of biologically synthesized nanocubes in wastewater treatment was done. A filter was designed. The colloidal solution of silver nanoparticles was mixed with activated charcoal in ratio of 1:1, 1:2 and 2:1 respectively, to make filter bed. A series of microbiological tests carried out on the filtrates obtained, showed that the microbes were killed to an extent of $97 \%$, especially $E$. coli. Our work indicates that silver nanoparticle loaded activated charcoal filter can be used as an excellent antibacterial water filter.
\end{abstract}

Keywords: Statistical optimization; Silver nanocubes; Green synthesis; Water treatment; Activated charcoal

\section{Introduction}

Metallic nanoparticles have generated a lot of attraction and are being investigated thoroughly nowadays due to various applications in the fields of biosensors, biomedicine, and biotechnology. They have unique size dependent properties due to large surface to volume ratio. Synthesis of silver nanoparticles particularly has been the focus of research because of its antimicrobial properties which have been known since ages. Various methods for synthesis of silver nanoparticles have been used including physical, chemical methods [1-3]. However these methods include use of toxic chemicals and generation of hazardous by products [4]. Therefore, synthesis via biological route is increasingly used now-a-days due to its cost effectiveness, simplicity, ecofriendly and nontoxic approach. Plant extract mediated synthesis has been reported for various species like Pelargonium graveolens, its endophytic fungus Colletratrichum sp. [5] Hibiscus rosa sinensis [6] and Coriander [7]. But this particular biological method is yet to be explored fully. Citrus limetta is small tree which may reach $8 \mathrm{~m}$ in height. It has irregular branches, and relatively smooth, brownishgrey bark. Citrus species contain a wide range of active ingredients such as vitamin C, flavanoids etc. [8,9]. Apart from this Citrus species are rich in Citric acid and Ascorbic acid. Research is going on to find their role as a reductant in synthesis of silver nanoparticles. Quite a few studies are available that report the use of Citrus sp. as effective natural, renewable and low-cost bioreducing agent for the synthesis of silver nanoparticles. Also, recently it has been reported that citrus peel yields thousands fold more phenolic compound than pulp. In India, about 2.15 million tons of citrus peel out of 6.28 million tons of citrus fruits is produced annually [10]. Citrus peel represents an economic and cost effective substrate for green synthesis of silver nanoparticles. In this study, we have worked with peels of Citrus limetta. Extract was prepared from Citrus limetta peel which acts as a bioreducing agent for silver nitrate. The yield of biological method of synthesis of silver nanoparticles is low. So optimization is done to get a significant yield. Shape and size of synthesized nanoparticles can be controlled by modulating temperature and $\mathrm{pH}$ [11]. Nanoparticles synthesis depends on interaction of various factors such as concentration of $\mathrm{AgNO}_{3}$ (substrate), volume of reductant, $\mathrm{pH}$, temperature etc. So careful optimization of different factors should be done and their interaction should be determined for effective synthesis of silver nanoparticles. Plackett Burman Design is used to determine the interaction and to find out the best among all the factors involved. In our design we have considered six independent variables. Silver nanocubes have been reported in a very few studies till now. It has been reported by Chen et al. that silver nanocubes have a higher antimicrobial activity than silver nanoplates or silver nanospheres [12]. Also silver nanocubes have various applications in the field of photonics, catalysis, SERS-based sensing [13]. Here we have reported the synthesis of silver nanocubes from previously unexplored statistically optimized bioreduction of silver nitrate using Citrus limetta peels as a substrate.

\section{Materials and Methods}

The Citrus limetta were collected from local market located at Vellore, India. Silver Nitrate $\left(\mathrm{AgNO}_{3}, 99.9 \%\right.$ pure) was obtained from Sisco Research Lab Pvt. Ltd, Mumbai. All chemicals were used without any further purification or treatment. All experiments were done in duplicates. Double distilled water was used for all the experiments.

\section{Preparation of the extract}

For synthesis fresh peels of Citrus limetta were collected, washed thoroughly with double distilled water and chopped into small pieces. $4 \mathrm{~g}$ of this finely cut Citrus limetta peels were weighed and transferred

*Corresponding author: Priyanka Srivastava, Assistant Professor, Division of Biomedical Sciences, School of Bio Sciences and Technology, VIT University, Vellore 632014, Tamil Nadu, India, Tel: +91 8870514196; E-mail: priyanka@vit.ac.in

Received July 11, 2014; Accepted August 27, 2014; Published September 02 2014

Citation: Trivedi P, Khandelwal M, Srivastava P (2014) Statistically Optimized Synthesis of Silver Nanocubes from Peel Extracts of Citrus limetta and Potential Application in Waste Water Treatment. J Microb Biochem Technol S4: 004. doi:10.4172/1948-5948.S4-004

Copyright: $\odot 2014$ Trivedi $P$, et al. This is an open-access article distributed under the terms of the Creative Commons Attribution License, which permits unrestricted use, distribution, and reproduction in any medium, provided the original author and source are credited 
into a $250 \mathrm{ml}$ beaker containing $40 \mathrm{ml}$ double distilled water, mixed well and boiled for $20 \mathrm{~min}$. The extract obtained was filtered through Whatman No. 1 filter paper and the filtrate was collected and stored for further use.

\section{Synthesis of silver nanoparticles}

Plackett Burman design was used to obtain optimized synthesis of silver nanoparticles. Six factors (Concentration of $\mathrm{AgNO}_{3}$, Volume of reductant, $\mathrm{pH}$, temperature, reaction volume and illumination) were used for analysis. All factors were tested in duplicates (S-1 to S-24). The upper and lower limits of experimental factors were determined in preliminary experiments. Aqueous solution of silver nitrate $\left(\mathrm{AgNO}_{3}\right)$ was prepared using double distilled water with constant stirring. Citrus peel extract was added to $\mathrm{AgNO}_{3}$ solution and stirred continuously. Color change was observed and OD was taken at wavelength range of 350-600 nm in UV-Visible Spectrophotometer. After stabilization of reaction of silver nanoparticle solution, pellet was prepared. For pellet preparation, $10 \mathrm{ml}$ of silver nanoparticles solution was taken in falcon tubes and centrifuged at $10000 \mathrm{rpm}$ for $15 \mathrm{~min}$. Pellet was thoroughly washed 3 times with double distilled $\mathrm{H}_{2} \mathrm{O}$ and centrifuged for $10 \mathrm{~min}$ at same speed. Pellet was dried in hot air oven at $40^{\circ} \mathrm{C}$. The yield for all the samples was calculated using the standard equation $y=0.327 x+0.068$, where $\mathrm{y}$ is the $\mathrm{OD}$ and $\mathrm{x}$ is the concentration.

\section{Characterization}

UV-Vis spectroscopy: The Ag nanoparticles were characterized in a HITACHI-U $2800 \mathrm{UV}-\mathrm{Vis}$ Spectrophotometer. The scanning range for the samples was $350-600 \mathrm{~nm}$. Base line correction of the spectrophotometer was carried out by using a blank reference. The prepared aqueous solutions (S-1 to S-24) of Ag nanoparticles showed an absorption band at $390-450 \mathrm{~nm}$, which is a typical absorption band of Ag nanoparticles due to their surface plasmon.

Scanning electron microscopy: Scanning Electron Microscopic (SEM) analysis was done using FEI Quanta FEG 200-High Resolution Scanning Electron Microscope. Thin films of the sample were prepared on a copper grid by just dropping a very small amount of the sample on the grid; extra solution was removed using a blotting paper. The sample was sputter coated with gold and analyzed.

\section{Plackett burman design}

A six factor Plackett Burman design was used to study the effect of variables on synthesis of silver nanoparticles. Variables chosen were temperature $\left(27^{\circ} \mathrm{C}, 60^{\circ} \mathrm{C}\right), \mathrm{pH}(6,8.5)$, volume of reductant $(3 \mathrm{ml}, 15$ $\mathrm{ml})$, volume of reaction vessel $(250 \mathrm{ml}, 1000 \mathrm{ml})$, illumination (dark, light) and concentration of silver nitrate $(0.001 \mathrm{M}, 0.01 \mathrm{M})$. They were designated as A, B and C, D, E, F respectively and values in brackets represent lower (-) and upper limit $(+)$ of each variable. The upper and lower limits for analysis were decided after performing a set of 14 preliminary experiments. Analysis was done using Minitab 16 software which generated a 24 run table for 6 factors. All experiments were performed in duplicates to minimize experimental errors.

\section{Designing of silver nanoparticle loaded activated carbon matrix filter for wastewater treatment}

Water filter was designed keeping in mind it should be cheap and effective. Food grade waste plastic bottles and cola tins were used. Filter had two chambers: upper and lower (Figure 1). Upper chamber stored crude/contaminated water which opened into the lower chamber containing AgNPs: AC filter bed of varying concentrations vis. 1:2, 1:1

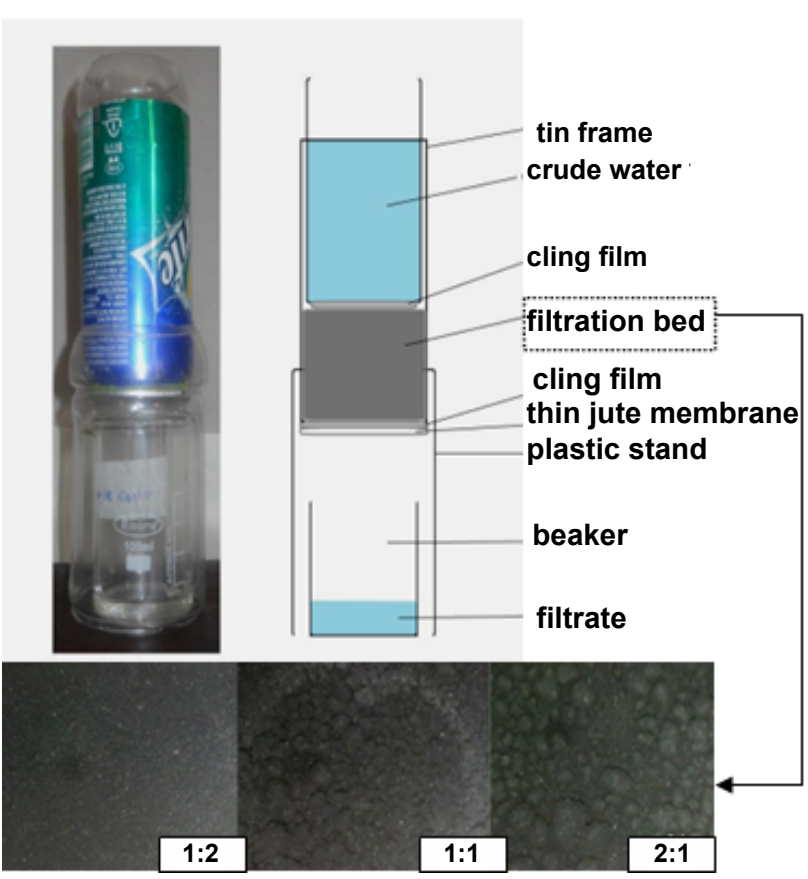

Figure 1: Design of silver nanoparticle loaded activated carbon matrix filter for wastewater treatment. Arrow represents filter bed with AgNPs:AC in 1:2, $1: 1$ and $2: 1$ ratio

and 2:1. Filter bed was prepared by adding $20 \mathrm{~g}$ activated charcoal to $10 \mathrm{ml}, 20 \mathrm{ml}$ and $40 \mathrm{ml}$ of AgNPs colloidal solution with continuous stirring the mixture. After that mixture was kept in sonicating water bath (The ILO Co., India) for $1 \mathrm{~h}$ and then allowed to air dry (Figure 1). Both chambers were separated by primary filter made up of a perforated cling film. Sediments in contaminated water were filtered while passing through primary filter. Secondary filter was designed using cling film with tiny pores on the inner side and thin jute membrane on the outer side. Contaminated water was purified while passing through AC-AgNPs filter bed, due to high surface area of activated charcoal, which aided in accelerated adsorption process and also due to the antimicrobial activity of silver nanoparticles. Filtrate was collected in $100 \mathrm{ml}$ beaker. Filtration process was solely gravity dependent, no pumps were used.

Antibacterial efficiency of AgNP:AC filter bed: Spread plate technique was used for primary microbiological characterization of filtrate. Nutrient agar $(2.3 \mathrm{~g})$ and agar $(1 \mathrm{~g})$ were added to $100 \mathrm{ml}$ distilled water and mixed well. After sterilization of media, $20 \mathrm{ml}$ of nutrient media was poured in each of four petriplates and allowed to solidify. Primary microbiological characterization was done for positive control, crude sample and three different filtrate samples obtained from AgNPs:AC-1:2, AgNPs:AC-1:1, AgNPs:AC-2:1 respectively. 100 $\mu \mathrm{l}$ each of water sample for positive control, crude sample and three filtrate samples were spread on the plate. For negative control only nutrient agar media was used, no plating of water samples was done. For crude, contaminated waste water was used for plating. For positive control, filtrate from only activated charcoal filter bed was used (no AgNPs in filter bed for positive control).Water samples were spread on the solidified medium. The plates were observed after incubation of six 6 hours and colonies were counted using android based 'colony counter'. All experiments were done in triplicates.

DNA estimation of water samples by Diphenylamine method: 
Citation: Trivedi P, Khandelwal M, Srivastava P (2014) Statistically Optimized Synthesis of Silver Nanocubes from Peel Extracts of Citrus limetta and Potential Application in Waste Water Treatment. J Microb Biochem Technol S4: 004. doi:10.4172/1948-5948.S4-004

DNA estimation of water samples was done following the standard procedure of diphenylamine method [14]. A fresh solution of diphenylamine solution was prepared by dissolving $1 \mathrm{~g}$ of diphenylamine (Nice chemicals, India) in $100 \mathrm{ml}$ of glacial acetic acid (Sigma Aldrich, India) and $2.5 \mathrm{ml}$ of concentrated $\mathrm{H}_{2} \mathrm{SO}_{4}$ (Sigma Aldrich, India). $2 \mathrm{ml}$ of DPA reagent was added to all standard and test samples. The contents of the tubes were mixed by vortexing. Test tubes were then incubated on a boiling water bath for 10 mins. Absorbance was measured at 595 $\mathrm{nm}$ against blank. Standard curve was plotted by taking concentration of DNA along $\mathrm{X}$-axis and absorbance at $595 \mathrm{~nm}$ along $\mathrm{Y}$-axis. From
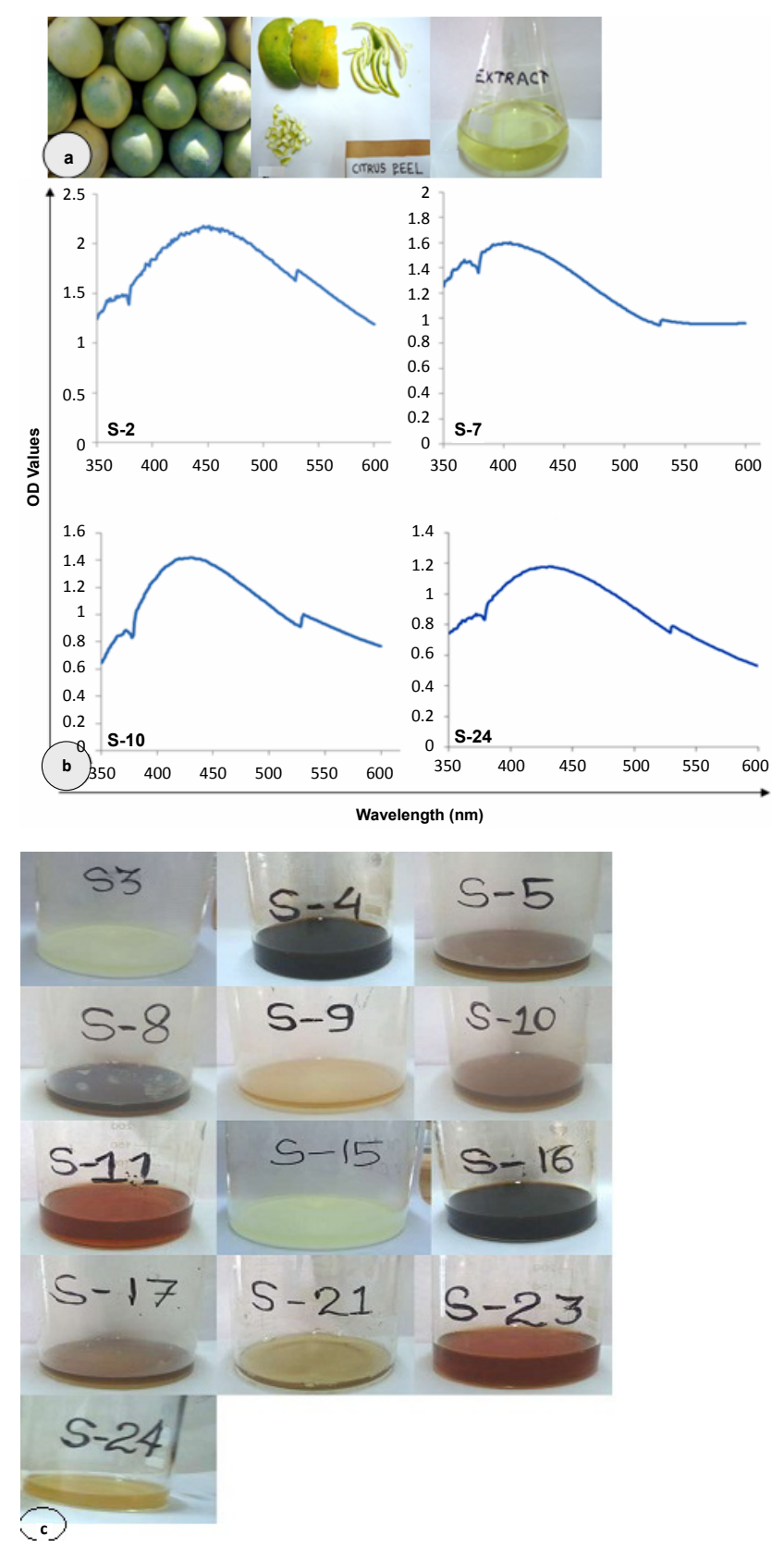

Figure 2: (a) Bioreductant used for study Citrus limetta, its peel and derived extract (b) Representative UV spectra showing synthesis of silver nanoparticles at different reaction conditions utilizing peel extract as the source of reductant (c) Color change in different samples after addition of peel extract.

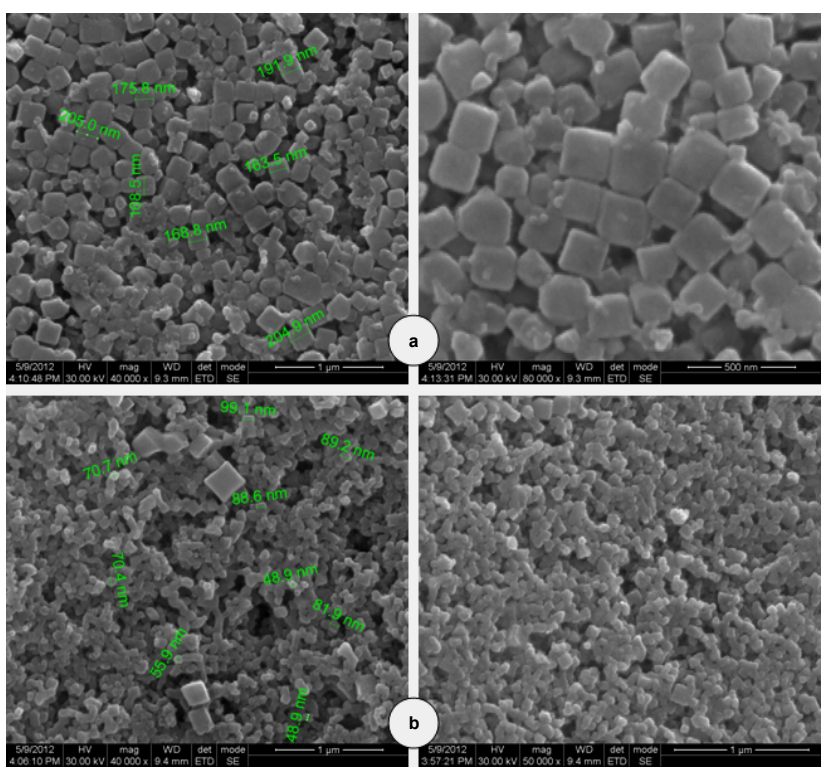

Figure 3: SEM micrograph of silver nanoparticles from two samples (a) S-24, (b) S-10.

this standard curve, the concentration of DNA in the given samples was calculated.

Quantitave analysis of carbohydrate using Benedict's assay: Benedict's reagent was prepared by following the standard method [15]. Standard glucose solution (1\%) was prepared. For crude (S1), 10 ml crude sample was mixed with $40 \mathrm{ml}$ of $1 \%$ glucose solution. Positive control (S2) was prepared by mixing $10 \mathrm{ml}$ positive control to $40 \mathrm{ml}$ of $1 \%$ glucose solution. For filtrate (S3), $10 \mathrm{ml}$ filtrate is mixed with $40 \mathrm{ml}$ of $1 \%$ glucose solution.

Screening of $\boldsymbol{E}$. coli in filtrate by using MacConkey agar plate: MacConkey agar (1.5 g) and agar ( $1 \mathrm{~g}$ ) were added in $200 \mathrm{ml}$ of distilled water in a $500 \mathrm{ml}$ conical flask. Media was sterilized and after setting of media in petriplates, different samples of filtrate, crude water, positive control and pure E. coli strain as control were streaked over the plates. The petridishes were incubated at $37^{\circ} \mathrm{C}$ for $18-20$ hours.

\section{Results and Discussions}

\section{Synthesis of silver nanoparticles}

Nanoparticles were synthesized from peel of fresh and healthy fruits of Citrus limetta (Figure 2a). After the addition of peel extract to silver nitrate solution, the color changed from pale yellow to brownish indicating the synthesis of silver nanoparticles. The color change was due to the excitation of surface plasmon vibrations with the silver nanoparticles [16].Similar observations were made during green synthesis of silver nanoparticles using the extracts of Citrus lemon [17].

Different color changes were observed for various samples depending on the conditions they were subjected to (Figures $2 b$ and 2c). It was observed that increase in temperature increases the rate of reaction and consequently faster color change of reaction mixtures. Also, with the addition of $\mathrm{NaOH}$ to the reaction mixtures, color change was very fast. We observed that a combination of high reductant volume, high temperature and high amount of $\mathrm{NaOH}$ resulted in abrupt color change for S-8 and S-20. This abrupt color change was also observed by Sahoo et al. [18] Color change was observed to be slow 
Citation: Trivedi P, Khandelwal M, Srivastava P (2014) Statistically Optimized Synthesis of Silver Nanocubes from Peel Extracts of Citrus limetta and Potential Application in Waste Water Treatment. J Microb Biochem Technol S4: 004. doi:10.4172/1948-5948.S4-004

in S-12 and S-24 and reaction was completed in 96 hours. For all other samples, reaction started 10-30 minutes after incubation.

\section{Characterization}

UV-Vis spectroscopy: The prepared aqueous solutions (S-1 to S-24) of Ag nanoparticles showed an absorption peak in the range of 390-450 $\mathrm{nm}$, which is a typical absorption band of silver nanoparticles. It is well recognized that the absorbance of Ag NPs mainly depends upon the size and shape [19]. We observed a sharp narrow peak for S-24 and S-10 indicating towards the formation of monodisperse nanoparticles. Different patterns of absorption were observed in different samples (Figure 2b). Good stability, reproducibility and nature of peak in UVvisible spectroscopy led us to select samples S-24 and S-10 for further studies.

SEM analysis: The SEM image of silver nanocubes synthesized by biological reduction method and green synthesis process by using Citrus limetta peel extract $\mathrm{AgNO}_{3}$ was shown in Figure $3 \mathrm{a}$ and $\mathrm{b}$. The SEM image show clear synthesis of nanocubes. The reaction conditions for S-10 and S-24 are almost similar (Volume of reductant: $3 \mathrm{ml}, \mathrm{pH}$ 6, temperature: $27^{\circ} \mathrm{C}$ ). The main difference lies in concentration of $\mathrm{AgNO}_{3}$ which is $0.001 \mathrm{M}$ for S-24 and $0.01 \mathrm{M}$ for S-10.

Size and shape of nanocubes was uniform in S-24 as compared to S-10, where some aggregation was also observed. Figures indicate that nanoparticles were more stable in S-24 while in S-10 some aggregation was observed. In S-24, nanoparticles were in the range of 163-205 nm, and this range was lower in S-10 being 37-59 nm. Nanocubes have a higher peak absorbance as seen in S-24, while aggregated nanoparticles have lower absorbance maxima as seen in S-10. Reason of selecting these two samples for analysis was significant yield and stability.

\section{Plackett burman statistical analyses}

The upper and lower limits for the parameters were fixed on the basis of preliminary experiments. A 24-run table was generated by
Minitab 16 statistical software, from the six variables considered for this study (Table 1). It was observed that temperature was the most significant factor that affected the synthesis of silver nanoparticles, followed by illumination and $\mathrm{pH}$. Concentration of silver nitrate was the least significant factor (Figure 4). Using the standard equation mentioned in material and methods section, yields for different samples were calculated and listed in (Figure 4).

\section{Antibacterial efficiency of AgNP: AC filter bed}

A higher number of colonies were observed in crude ie. the contaminated water sample (177) and positive control i.e. filtrate obtained by using only activated charcoal as filter bed (148) as compared to filtrates. In their case, number of microbial colonies decreased with increase in concentration of AgNPs (65 in 1:2, 13 in 1:1 and 6 in 2:1 of AgNP:AC), respectively. Phong et al. [20] reported 100\% antibacterial efficiency of Ag nanoparticle filter for gram negative bacteria, such as $E$. coli and about $99.79 \%$ antibacterial efficiency in gram positive bacteria. In the present study, we have reported antimicrobial efficiency of $96.6 \%$ and $100 \%$ antibacterial efficiency against gram negative bacteria (E. coli). This efficiency can be further increased by incorporating other techniques in silver nanoparticle based filters such as use of ultraviolet radiation, ultrasonication and antimicrobial agent. Water filters with such new approaches show drastic increase in effectiveness in contaminated water purification process [21].

\section{Estimation of DNA in different samples of filtrate by using Diphenylamine method}

The basic idea behind this estimation was that more amount of DNA would be present in sample with higher number of microbes and vice versa. Standard graph was plotted by using O.D. values measured using spectrophotometer at $595 \mathrm{~nm}$. From the standard graph, DNA concentration in crude sample was found to be $5.308 \mu \mathrm{g} / \mathrm{ml}$ and in positive control it was $4.842 \mu \mathrm{g} / \mathrm{ml}$. Treated filtrate showed DNA concentration of $1.751 \mu \mathrm{g} / \mathrm{ml}$ (in 1:2), $1.673 \mu \mathrm{g} / \mathrm{ml}$ (in 1:1) and 1.596

\begin{tabular}{|c|c|c|c|c|c|c|c|}
\hline Std Order & Run Order & Conc of $\mathrm{AgNO}_{3}(\mathrm{M})$ & Volume of reductant (ml) & $\mathbf{P h}$ & Tecmperature $\left({ }^{\circ} \mathrm{C}\right)$ & Reaction Volume (ml) & Illumination \\
\hline 1 & 1 & 0.01 & 3 & 9 & 27 & 250 & Dark \\
\hline 15 & 1 & 0.001 & 15 & 9 & 27 & 1000 & Dark \\
\hline 19 & 1 & 0.001 & 15 & 9 & 80 & 250 & Light \\
\hline 5 & 1 & 0.01 & 15 & 6 & 80 & 1000 & Dark \\
\hline 21 & 1 & 0.001 & 3 & 6 & 80 & 1000 & Light \\
\hline 6 & 1 & 0.01 & 15 & 9 & 27 & 1000 & Light \\
\hline 13 & 1 & 0.01 & 3 & 9 & 27 & 250 & Dark \\
\hline 11 & 1 & 0.001 & 15 & 6 & 27 & 250 & Light \\
\hline 3 & 1 & 0.001 & 15 & 9 & 27 & 1000 & Dark \\
\hline 8 & 1 & 0.001 & 3 & 9 & 80 & 1000 & Dark \\
\hline 7 & 1 & 0.001 & 15 & 9 & 80 & 250 & Light \\
\hline 9 & 1 & 0.001 & 3 & 6 & 80 & 1000 & Light \\
\hline 24 & 1 & 0.001 & 3 & 6 & 27 & 250 & Dark \\
\hline 10 & 1 & 0.01 & 3 & 6 & 27 & 1000 & Light \\
\hline 14 & 1 & 0.01 & 15 & 6 & 80 & 250 & Dark \\
\hline 16 & 1 & 0.01 & 3 & 9 & 80 & 250 & Light \\
\hline 18 & 1 & 0.01 & 15 & 9 & 27 & 1000 & Light \\
\hline 4 & 1 & 0.01 & 3 & 9 & 80 & 250 & Light \\
\hline 17 & 1 & 0.01 & 15 & 6 & 80 & 1000 & Dark \\
\hline 23 & 1 & 0.001 & 15 & 6 & 27 & 250 & Light \\
\hline 2 & 1 & 0.01 & 15 & 6 & 80 & 250 & Dark \\
\hline 22 & 1 & 0.01 & 3 & 6 & 27 & 1000 & Light \\
\hline 20 & 1 & 0.001 & 3 & 9 & 80 & 1000 & Dark \\
\hline
\end{tabular}

Table 1: Plackett Burman analysis using Minitab 16 generated a 24 run table for 6 factors; all experiments were done in duplicates. 


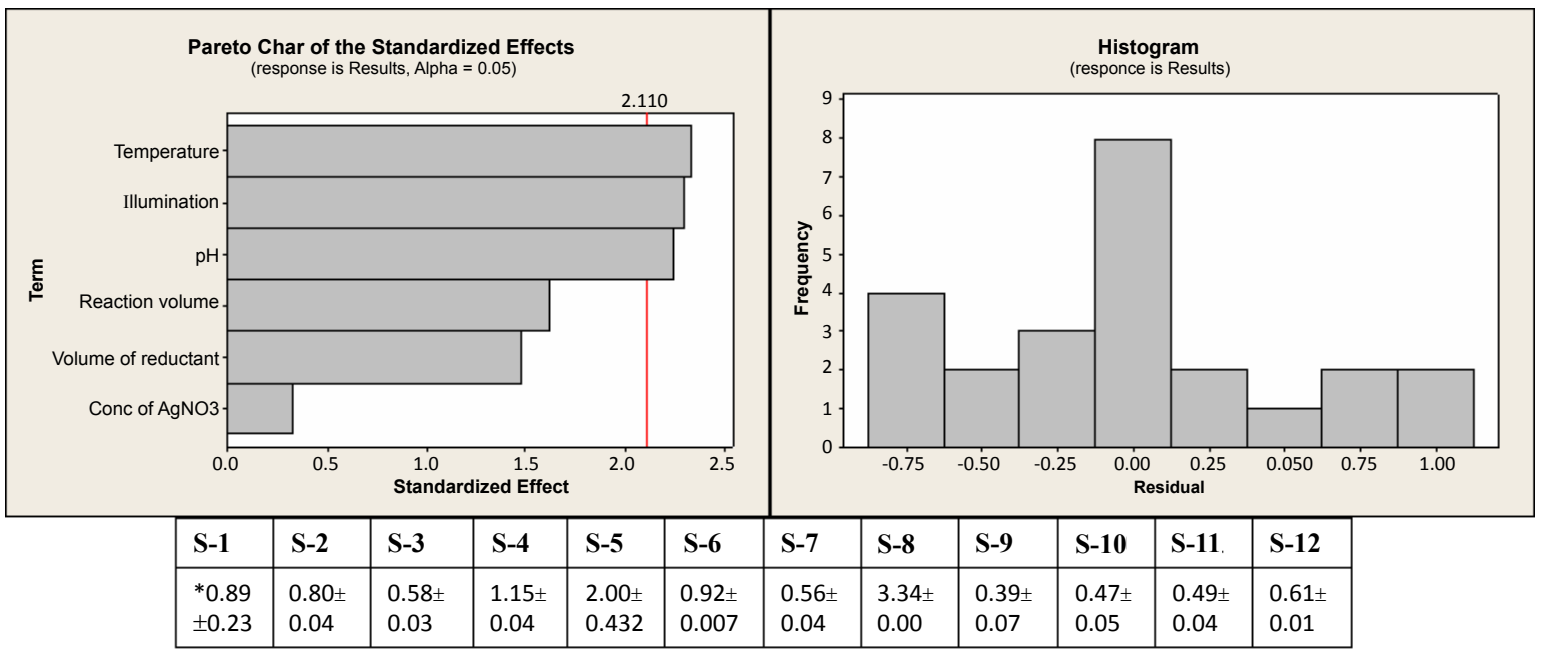

Figure 4: Graph showing significant factors affecting Ag nanoparticle synthesis and frequency of residual values (Expected-Observed).
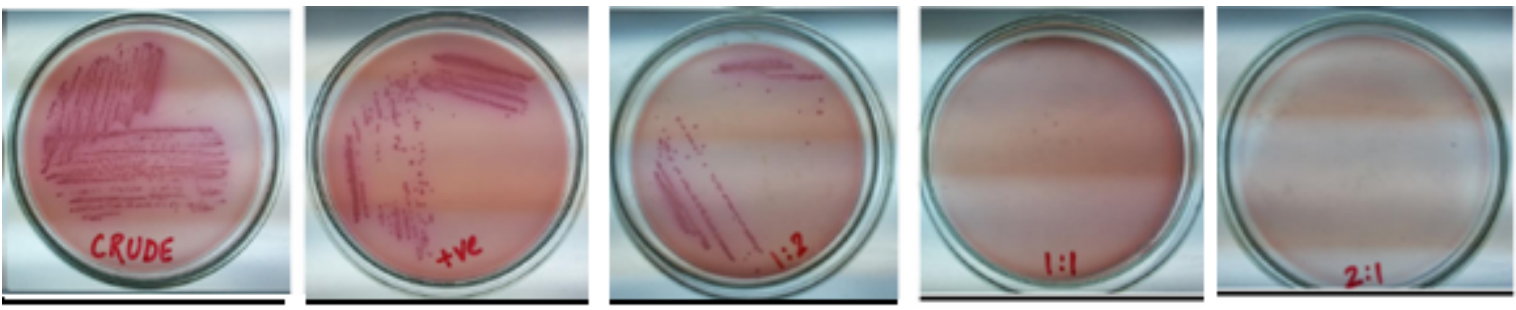

Figure 5: MacConkey agar plating showing colonies of E. coli in contaminated water sample (crude), positive control (activated charcoal filter bed) and filter bed containing different ratios of AgNPs with activated charcoal (AC).

$\mu \mathrm{g} / \mathrm{ml}$ (in 2:1) of AgNPs: AC, respectively. Thus, we can conclude that the number of microbes decreased with the increase in AgNP concentration in AgNP:AC filter.

\section{Quantitative analysis of different filtrate samples by Benedict's carbohydrate assay}

Carbohydrate assay was done on the different samples of filtrate. The results shows amount of dextrose degraded in 24 hours. It was found to be $0.207 \mathrm{~g}$ for crude water, $0.155 \mathrm{~g}$ for positive control and $0.124 \mathrm{~g}$ for filtrate (1:1; AgNPs: AC). So, more the number of microbes more will be carbohydrate degradation. A decrease in degraded carbohydrate concentration in case of $(1: 1$; AgNPs: AC) shows that the microbial count has decreased due to antimicrobial effect of silver nanoparticles. Similar implications have been drawn by Burney et al. [22].

\section{MacConkey agar plating}

MacConkey agar plating is done for isolation of enteric Gram negative bacteria. Pink color colonies show presence of $E$. coli [23]. Pink colonies (E. coli) were not observed on plates, where 1:1 and 2:1 filtrates were plated showing $100 \%$ effectiveness of filter bed against gram negative bacteria (E. coli). Few bacterial colonies could be seen on low concentration silver nanoparticle filter bed filtrate (1:2 AgNPs:AC), however with increase in concentration of silver nanoparticles in filter bed, total number of bacterial colonies decreased to none in filtrate (Figure 5).

\section{Conclusions}

We were successfully able to synthesize silver nanocubes from the peel of Citrus limetta. As mentioned in the introductory section, this is one of the most feasible sources of reductant due to its low cost and all year round availability in Indian context. Apart from this, the work reported here stands out from others in various aspects. First, this is the first report which calculates the yield of synthesized AgNPs from a biological substrate. Yield is an important indicator to demonstrate the efficiency of used substrates and reductants in a reaction. Secondly, conditions for optimal yield of AgNPs were screened using Plackett Burman design. To the best of our knowledge this is the first study that has reported synthesis of silver nanocubes from Citrus limetta peel as substrate. In terms of yield, S-10 and S-24 were best and the particles were in narrow size range and stable as confirmed by the results of SEM. Fourth important highlight of this study is use of silver nanocubes with activated carbon for waste water treatment. A water filter was designed and microbiological characterization of filtrate was done. DNA estimation, Benedict's carbohydrate assay and MacConkey agar plating of filtrate suggested that the antimicrobial action showed a considerable improvement with increase in concentration of the silver nanoparticles in the filter bed.

\section{References}

1. Nair LS, Laurencin CT (2007) Silver nanoparticles: synthesis and therapeutic applications. J Biomed Nanotechnol 3: 301-316.

2. Zhang $Y$, Peng H, Huang W, Zhou Y, Yan D (2008) Facile preparation and characterization of highly antimicrobial colloid Ag or Au nanoparticles. J Colloid Interface Sci 325: 371-376. 
Citation: Trivedi P, Khandelwal M, Srivastava P (2014) Statistically Optimized Synthesis of Silver Nanocubes from Peel Extracts of Citrus limetta and Potential Application in Waste Water Treatment. J Microb Biochem Technol S4: 004. doi:10.4172/1948-5948.S4-004

3. Sharma VK, Yngard RA, Lin Y (2009) Silver nanoparticles: green synthesis and their antimicrobial activities. Adv Colloid Interface Sci 145: 83-96.

4. Thakkar KN, Mhatre SS, Parikh RY (2010) Biological synthesis of metallic nanoparticles. Nanomedicine 6: 257-262.

5. Mukherjee P, Ahmad A, Mandal DS, Senapati S, Sainkar R, et al. (2001) Fungus-mediated synthesis of silver nanoparticles and their immobilization in the mycelial matrix: a novel biological approach to nanoparticle synthesis. Nano Letts 1: 515 .

6. Philip D (2010) Green synthesis of gold and silver nanoparticles using Hibiscus rosa sinensis. Physica E: Low-dimensional Sys Nanostruct 42: 1417-1424.

7. Narayanan KB, Sakthivel N (2008) Coriander leaf mediated biosynthesis of gold nanoparticles. Mater Letts 62: 4588-4590.

8. Li S, Lambros T, Wang Z, Goodnow R, Ho CT (2007) Efficient and scalable method in isolation of polymethoxyflavones from orange peel extract by supercritical fluid chromatography. J Chromatogr B Analyt Technol Biomed Life Sci 846: 291-297.

9. Guimarães R, Barros L, Barreira JC, Sousa MJ, Carvalho AM, et al. (2010) Targeting excessive free radicals with peels and juices of citrus fruits: grapefruit, lemon, lime and orange. Food Chem Toxicol 48: 99-106.

10. Kaur A, Singh S, Singh RS, Schwarz WH, Puri M (2010) Hydrolysis of citrus peel naringin by recombinant a-L-rhamnosidase from Clostridium stercorarium. J Chem Technol Biotechnol 85: 1419-1422.

11. Gericke M, Pinches A (2006) Microbial production of gold nanoparticles. Gold Bull 39: 22-28

12. Chen E, Su H, Zhang W, Tan T (2011) A novel shape-controlled synthesis of dispersed silver nanoparticles by combined bioaffinity adsorption and TiO"2 photocatalysis. Powder Technol 212: 166-172.
13. Sun Y, Xia Y (2002) Shape-controlled synthesis of gold and silver nanoparticles. Science 298: 2176-2179

14. Burton K (1956) A study of the conditions and mechanism of the diphenylamine reaction for the colorimetric estimation of deoxyribonucleic acid. Biochem J 62 : 315-323.

15. Benedict SR (1909) A Reagent For the Detection of Reducing Sugars. J Bio Chem 5: 485-487.

16. Parashar UK, Saxena SP, Srivastava A (2009) A Bio inspired synthesis of silver nanoparticles. Dig J Nanomater Bios 4: 159-166.

17. Mulvaney $P$ (1996) Surface plasmon spectroscopy of nanosized metal particles. Langmuir 12: 788-800.

18. Sahoo PK, Kalyan Kamal SS (2009) Synthesis of Silver Nanoparticles using Facile Wet Chemical Route. Defence Sci J 59: 447-455.

19. Mock JJ, Barbic M, Smith DR, Shultz DA (2002) Shape effect in plasmon resonance of individual colloidal silver nanoparticles. J Chem Phys 116: 67556759.

20. Phong NTP, Thanh NVK, Phuong PH (2009) Fabrication of antibacterial water filter by coating silver nanoparticles on flexible polyurethane foams. J Phys 187.

21. Tiwari DK, Behari J, Sen $P$ (2008) Application of Nanoparticles in Waste Water Treatment. World Appl Sci J 3: 417-433.

22. Burne CM, Davis PG, Johnson KM (1982) Diel relationships of microbial trophic groups and in situ dissolved carbohydrate dynamics in the caribbean sea Marine Biol 3: 311-322.

23. Daby D, Turner J, Jago C (2002) Microbial and nutrient pollution of coastal bathing waters in Mauritius. Environ Int 27: 555-566.
This article was originally published in a special issue, Chemical Engineering Science handled by Editor. Dr. Tingyue Gu, Ohio University, USA 